\title{
SPATIAL MAPPING OF INTERICTAL EPILEPTIC DISCHARGES IN FMRI WITH TOTAL ACTIVATION
}

\author{
F. Işık Karahanoğlu ${ }^{1,2}$, Frederic Grouiller ${ }^{2}$, Cesar C. Gaudes ${ }^{2,3}$, Margitta Seeck ${ }^{4}$, Serge Vulliemoz ${ }^{4}$, Dimitri Van De Ville ${ }^{1,2}$ \\ ${ }^{1}$ Institute of Bioengineering, Ecole Polytechnique Fédérale de Lausanne (EPFL),Switzerland \\ ${ }^{2}$ Department of Radiology and Medical Informatics, University of Geneva (UNIGE), Switzerland \\ ${ }^{3}$ Basque Center on Cognition, Brain and Language (BCBL), Spain \\ ${ }^{4}$ Department of Neurology, University of Geneva (UNIGE), Switzerland
}

\begin{abstract}
During the monitoring of pharmacoresistant epilepsy patients prior to surgery, interictal epileptic discharges (IEDs) are analyzed to locate possible sources of epileptic activity. In order to compensate low spatial resolution of EEG, simultaneous EEG-fMRI recordings can be used. Conventional methods typically deploy an EEGinformed analysis of the fMRI data; i.e., EEG-derived IED onset timings are used to setup regressors for linear regression. Recently we have proposed a new fMRI analysis method, total activation (TA), which is able to deconvolve the underlying activity-inducing signal without prior information on the onset timing and duration of events. Here we demonstrate that TA can locate the epileptogenic regions from fMRI data. We compare and validate our results with conventional methods performed by experts prior to surgery.
\end{abstract}

Index Terms - fMRI BOLD, spatiotemporal regularization, deconvolution, sparsity, epilepsy

\section{INTRODUCTION}

Electroencephalography (EEG) is the most prominent method to diagnose and monitor epilepsy noninvasively [1]. EEG measures the electric potential induced due to the brain activity by several electrodes connected to the scalp. However, EEG alone lacks the spatial resolution to localize the sources, especially in deep brain structures; i.e., EEG source localization is an ill-posed inverse problem that requires additional assumptions on the source model.

Simultaneous recording of EEG and functional magnetic resonance imaging (FMRI) is proposed to overcome the localization problem since the EEG signals can be cleaned from the MR artefacts [1]. FMRI blood oxygenated level dependent (BOLD) signal reflects metabolic and hemodynamic changes following neuronal activity. FMRI's high spatial resolution provide additional information to be combined with EEG's monitoring process. However, EEG has mostly been the primary imaging method; i.e., EEG-derived IED onsets are used to set up regressors of a general linear model (GLM) for fMRI. Recently, some exploratory methods propose fMRI derived EEG methods such as independent component analysis [2].

With the advent of fast solutions for convex sparsity constraints, variational formulations have been proposed for fMRI deconvolution. Assuming a linear model for the hemodynamic system, referred to as hemodynamic response function (HRF), they impose temporal sparsity in the brain. Contrary to confirmatory methods such as the GLM, none of these methods need timing information of

This work was supported in part by the Swiss National Science Foundation (under grants PP00P2-123438, 310030-132952, 122073, 141165, SPUM 140332) and in part by Center for Biomedical Imaging (CIBM) of the Geneva-Lausanne Universities and the EPFL. the paradigm, therefore, is able to detect spontaneous brain activity. For example [3] use the majorization-minimization scheme, [4] relies on ridge-regression, and [5] imposes sparsity on a dictionary of shifted HRFs whereas [6] imposes sparsity in the activelet-domain, a wavelet basis that is tailored to HRF. However, these methods only operate in the temporal domain and do not incorporate any spatial characteristics of fMRI. Moreover, variational formulations are constructed based on a "synthesis prior", which is an explicit dictionary; e.g., activelets or shifts of canonical HRF. We recently proposed a spatiotemporal regularization, namely total activation (TA), to derive the activity-inducing signal in fMRI [7]. Temporal regularization in TA employs an "analysis prior" with a general differential operator; i.e., inverting the HRF such that the operator can be easily tailored for the desired type of activity (spike-like or block-like) [8]. The spatial regularization imposes structured-sparsity based on an anatomical prior which accounts for similar patterns in a brain region. We employ the efficient generalized forward-backward splitting algorithm $[9,10]$, which is a fast iterative shrinkage algorithm that alternates between temporal and spatial domain solutions.

The detection of epileptogenic regions from fMRI constitutes a potential application for fMRI deconvolution methods since the onset timings of IEDs are not known a priori [11]. Here we show that TA reveals fMRI-derived IEDs contrary to conventional EEG derived methods. Specifically, we apply TA to an epilepsy patient data and correlate recovered activity-inducing signals with EEG-derived IED onsets. Then non-parametric hypothesis testing locate regions with epileptogenic activity. We compared and validated our results with the gold standard (GLM model) actually used by the experts prior to surgery [12].

\section{METHODS}

\subsection{FMRI Signal Model}

We represent the BOLD signal of $i^{t h}$ voxel as convolution of activity-inducing signal $u(i, t)$ with HRF $h(t)$. In real-world fMRI data, the BOLD signal is contaminated by different sources, such as movement, cardiac and respiratory fluctuations, scanner noise, nonhomogenous magnetic field [13]. Here, we presume that the data is preprocessed to compensate some of these effects including movement compensation, regression of low frequency drifts, and voxel-wise normalization (explained in section 3.1). The fMRI signal $y(i, t)$ then reduces to

$$
y(i, t)=u(i, t) * h(t)+\epsilon(i, t)=x(i, t)+\epsilon(i, t),
$$

where $x(i, t)$ is the activity-related signal, and $\epsilon(i, t)$ is independently distributed gaussian noise with zero mean and variance $\sigma_{i}^{2}$. We define the sparse innovation signal $u_{s}(i, t)$ from which activity- 
inducing signals are derived as $D\{u(i, \cdot)\}(t)=u_{s}(i, t)$, where $D$ is derivative operator. The innovation signal reflects onset and offset timings of neuronal events and conventional total variation regularization would favor block-like activity-inducing signals. Figure 1 (box I) illustrates the construction of fMRI signal from activityinducing signals.

\subsection{Hemodynamic Response Function}

Even though only empirical models exist for the HRF approximation, parametric estimations are widely employed in fMRI studies [14]. Here, we adopt the formulation in [6] based on the first-order Volterra series approximation of non-linear Balloon model. The authors derive a linear differential operator $L_{h}$, of which HRF $h(t)$ is the Green's function; i.e, $L_{h}\{h\}(t)=\delta(t)$. Specifically, the differential operator $L_{h}$ is defined by its zeros $\alpha_{i}(i=1, \ldots, 4)$ and pole $\gamma$ as follows

$$
L_{h}=\prod_{i=1}^{4}\left(D-\alpha_{i} I\right)(D-\gamma I)^{-1},
$$

where $I$ is the identity operator. The zeros and pole are set via the optimum parameter setting in [14]. The differential operator $L_{h}$ indeed inverts the effect of smooth hemodynamic system and uncovers the activity-inducing signal, $u$, and sparse innovation signal, $u_{s}$, as

$$
L_{h}\{x(i, \cdot)\}(t)=u(i, t), \quad L\{x(i, \cdot)\}(t)=u_{s}(i, t),
$$

where $L=D L_{h}$ being the general differential operator of TA.

From now on, we consider the discrete data, that is, noisy fMRI dataset is a matrix $\mathbf{y}=[y(i, t)]_{i, t}$ of size $V \times N$, where $i=$ $[1, \ldots, V]$ is the voxel index and $t=[1, \ldots, N]$ is the temporal index. All the operators are discretized, which we denote with $\Delta$; e.g., $\Delta_{L_{h}}$ indicates the difference operation for inverse HRF $L_{h}$.

\subsection{Total Activation}

TA aims at recovering underlying activity-inducing signals from noisy fMRI measurements through a convex problem imposing (structured)-sparsity priors in the spatiotemporal domain. Mainly, this variational formulation consists of least-squares data fitting term, a sparsity-promoting temporal regularization term which incorporates the general differential operator $L$, and a spatial regularization term which constructs structured-sparse patterns in the brain. Then we solve for

where

$$
\tilde{\mathbf{x}}=\arg \min _{\mathbf{x}} \frac{1}{2}\|\mathbf{y}-\mathbf{x}\|_{F}^{2}+\mathcal{R}_{T}(\mathbf{x})+\mathcal{R}_{S}(\mathbf{x}),
$$

and

$$
\mathcal{R}_{T}(\mathbf{x})=\sum_{i=1}^{V} \lambda_{1}[i] \underbrace{\left\|\Delta_{L}\{\mathbf{x}[i, \cdot]\}\right\|_{1}}_{\sum_{t=1}^{N}\left|\Delta_{L}\{x[i, t]\}\right|},
$$

$$
\mathcal{R}_{S}(\mathbf{x})=\sum_{t=1}^{N} \lambda_{2}[t] \underbrace{\left\|\Delta_{\text {Lap }}\{\mathbf{x}[\cdot, t]\}\right\|_{(2,1)}}_{\sum_{k=1}^{M} \sqrt{\sum_{i \in \mathrm{R}_{k}} \Delta_{\text {Lap }}\{x[i, t]\}^{2}}},
$$

$\|\mathbf{x}\|_{F}$ is the Frobenius norm and $\Delta_{\text {Lap }}$ is the Laplacian filter.

Temporal regularization term $\mathcal{R}_{T}(\mathbf{x})$ applies on each voxel time series and exploits the sparsity of innovation signal $\mathbf{u}_{\mathbf{s}}=\Delta_{L}\{\mathbf{x}\}$ imposing $\ell_{1}$-norm. On the other hand, the spatial regularization term $\mathcal{R}_{S}(\mathbf{x})$ acts on each fMRI volume and uses $\ell_{(2,1)}$-norm on brain parcels to enforce structured sparsity. Each parcel $R_{k}$ is based on an anatomical atlas with $M$ regions. We aim at recovering coherent activations inside regions whereas sparse activations across all volume. Regularization parameters $\lambda_{1}$ and $\lambda_{2}$ calibrates the influence of temporal and spatial constraints, respectively. TA flowchart is depicted in Figure 1 (box II).

\subsection{Optimization Algorithm}

We employ generalized forward-backward splitting [9], for denoising case also known as parallel Dykstra-like proximal algorithm [10], to solve the optimization problem in (3). The joint solution is obtained by incorporating the proximal maps of both spatial and temporal regularizations (Algorithm 1). The proximal map solves

$$
\operatorname{prox}_{\mathcal{R}_{T, S}}(\mathbf{y})=\arg \min _{\mathbf{x}} \frac{1}{2}\|\mathbf{y}-\mathbf{x}\|_{F}^{2}+\mathcal{R}_{T, S}(\mathbf{x}) .
$$

Since both regularization terms are convex but not quadratic, iterative methods are required. The temporal solution, $\operatorname{prox}_{\mathcal{R}_{T}}$, exploits generalization of total variation regularization introduced in [8] where a filter formulation of a discrete operator $\Delta_{L}=\Delta_{D} \Delta_{L_{h}}$ is constructed. We construct the minimum length filter beginning from the simple difference filter $\Delta_{D}=[1,-1]$. The analysis formulation in (4) provides serious advantages on the implementation of the difference filter; that is, the cascade filtering of a causal filter (length 6) for zeros $\left[0, \alpha_{1}, \ldots, \alpha_{4}\right]$ and either a causal filter $(\operatorname{Re}(\gamma)<0)$ or an anti-causal filter $(\operatorname{Re}(\gamma)>0)$. The spatial solution $\operatorname{prox}_{\mathcal{R}_{S}}$ is obtained via dual-norm formulation for $\ell_{(2,1)}$-norm [15] and gradient projection algorithm [16]. The denoising problem (Algorithm 1) solves for the activity-related signal $\tilde{\mathbf{x}}$, however, we can apply the differential operator $\Delta_{L_{h}}$ to recover the activity-inducing signal.

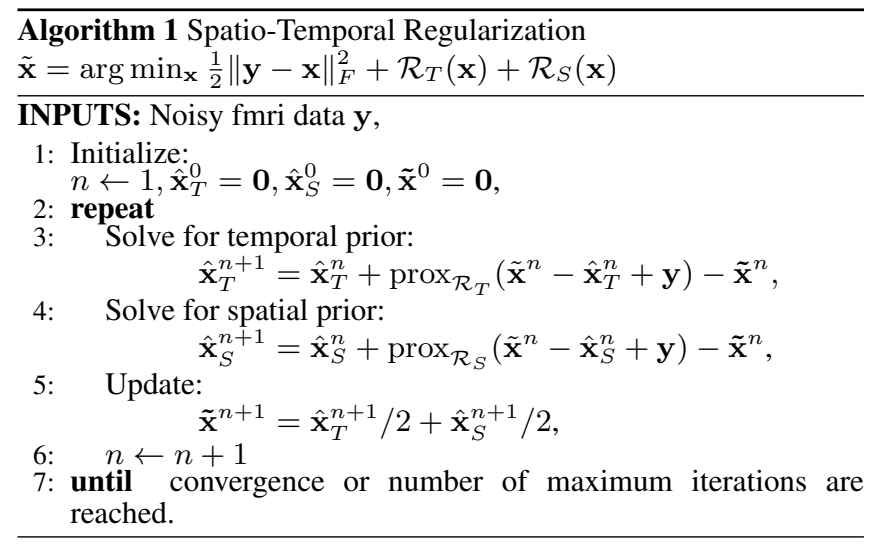

\section{RESULTS}

\subsection{EEG-FMRI Data}

One epilepsy patient was scanned with simultaneous EEG-fMRI. The fMRI data was acquired by a Siemens 3T Trio MR scanner with gradient echo EPI while resting (eyes-closed). The acquisition parameters were $\mathrm{TR}=1.5 \mathrm{~s}, \mathrm{TE}=35 \mathrm{~ms}$, flip angle $=85^{\circ}$, voxel size $=3.75 \times 3.75 \times 5.5 \mathrm{~mm}^{3}$, total 25 slices and 1100 scans. T1 and T2 weighted (pre \& post-operation) images were also acquired. In order to remove the artifacts prior to analysis, fMRI volumes were realigned to the first scan, spatially smoothed with Gaussian filter (FWHM=5mm) using SPM8 (FIL,UCL,UK). The anatomical AAL atlas [17] (90 regions without the cerebellum) was mapped onto the subjects functional space using the IBASPM toolbox [18]. The first 


\section{FMRI signal model}

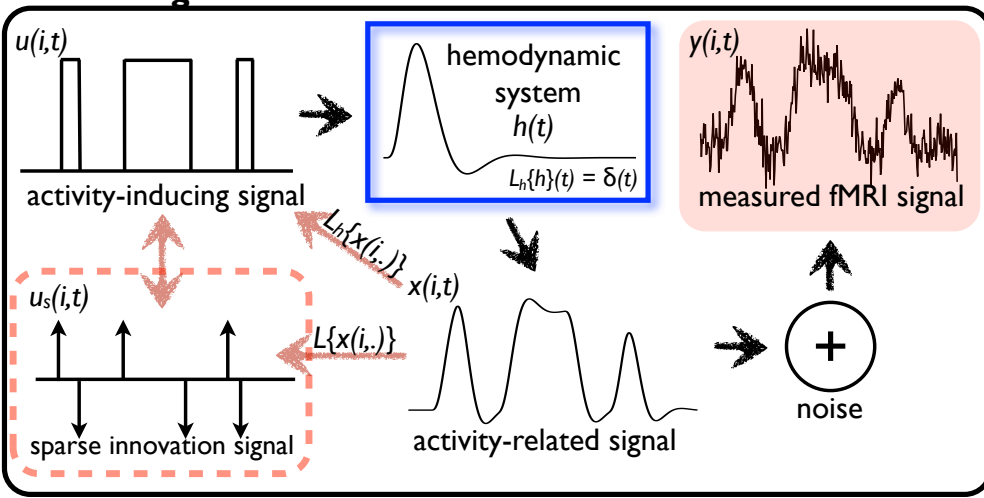

II. Total activation

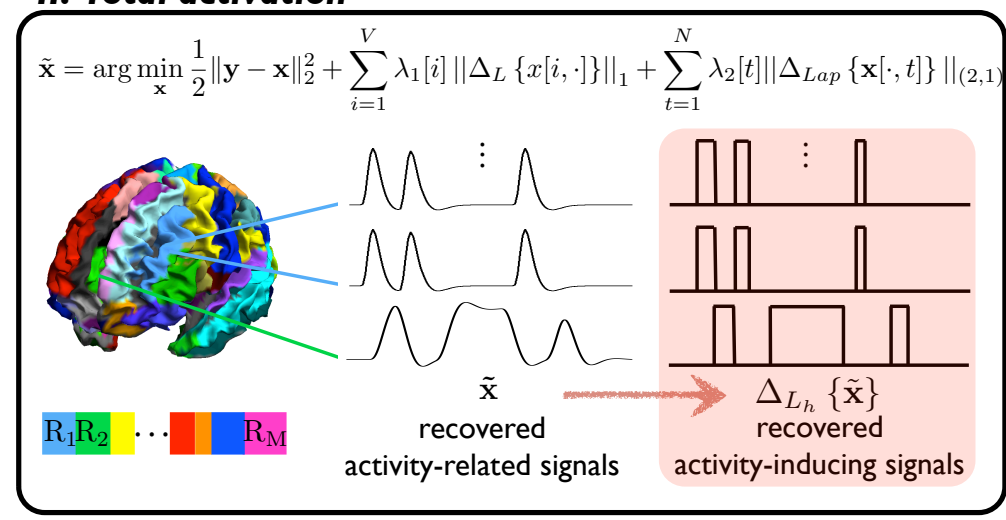

III. Non-parametric hypothesis testing

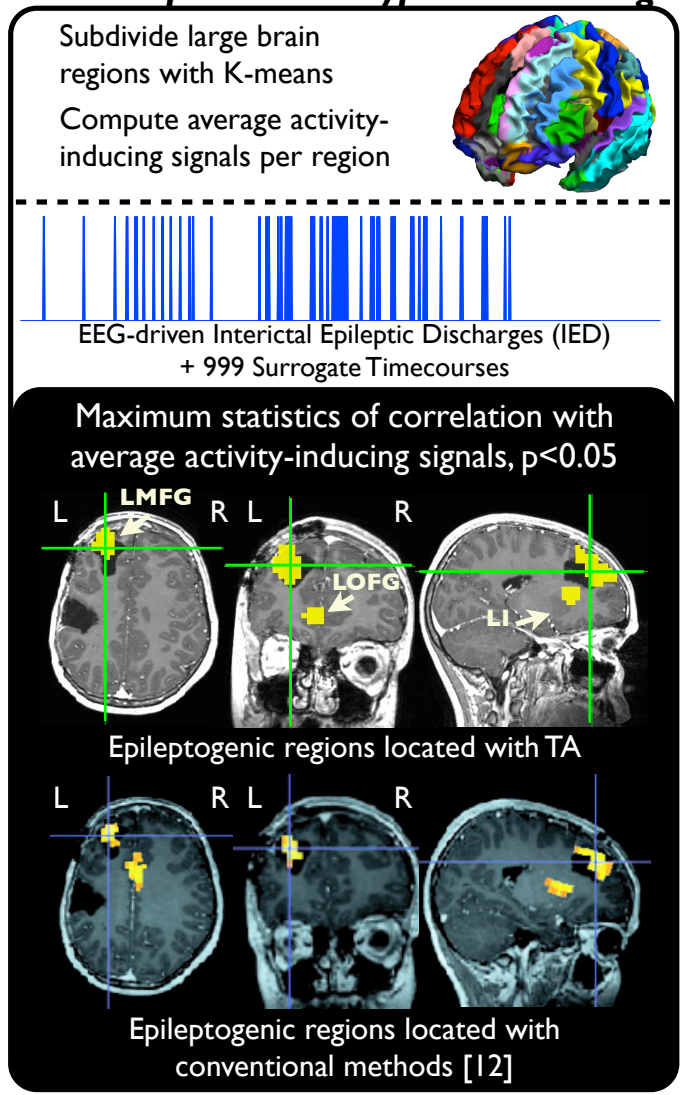

Fig. 1: Schematic of our approach for detecting IEDs from fMRI. Block-like activity-inducing signals are fed into the hemodynamic system which is formulated as the Green's function of a general derivative operator $L_{h}$; i.e., $L_{h}\{h\}(t)=\delta(t)$ (Box I). TA recovers neuronal-related activity-inducing signals by imposing sparsity constraints through spatiotemporal regularization (Box II). Nonparametric hypothesis testing revealed three significant regions, Left Middle Frontal Gyrus (LMFG) (resected area), Left Medial OrbitoFrontal Gyrus (LOFG), and Left Insula (LI) with $\mathrm{p}<0.05$ (corrected). The results are validated with conventional methods prior to surgery (Box III).

10 volumes are discarded and voxels time series labelled within the atlas were detrended for slow oscialltions using a first-degree polynomial and DCT basis function up to cut-off frequency of $1 / 125 \mathrm{~Hz}$, and finally scaled to have unit variance.

EEG signals were recorded with a 64 MR-compatible EEG cap (EasyCaps, FalkMinnow Services, Herrsching, Germany) according to the 10-20 system. Electrodes were equipped with an additional $5 \mathrm{k}$ resistance and impedances were kept as low as possible. EEG was acquired at $5 \mathrm{kHz}$ using 2 BrainAmp MR compatible amplifiers (Brain Products, Munich, Germany) and was synchronized with the MR clock. MR gradient and cardioballistic artefacts were removed from the EEG using Vision Analyzer (Brain Products, Munich, Germany) using average artifact subtraction methods [19]. EEG data was subsequently downsampled to $250 \mathrm{~Hz}$, and IED were visually marked by an experienced electroencephalographer.

\subsection{Localization of Interictal Epileptic Discharges with TA}

The algorithm is implemented in Matlab 7.14 (Mathworks, Natick, MA) on a 64-bit, 4-core computer with 16 GB RAM, operating Linux. The total allocated time was around 6 hours for data of size $(8598 \times 1090)$. Each loop in spatiotemporal regularization (Algorithm 1) includes two iterative proximal map computations defined in (6). The temporal regularization parameter $\lambda_{1}[i]$ is calibrated automatically in $\operatorname{prox}_{\mathcal{R}_{T}}$ such that the noise level converges to a preestimated noise level [20], derived from the median absolute deviation of fine-scale wavelet coefficients (Daubechies, order 3). We pick an empirical spatial regularization parameter $\lambda_{2}=5$ which seems to provide good compromise for data fitting. Increasing $\lambda_{2}$ forces the smoothness leaving no room for local differences, especially for big regions. On the other hand small $\lambda_{2}$ results in high variance in the regions. We refer to [7] for synthetic data results and more details. The recovered activity-inducing signals reflect not only IEDs but also spontaneous brain activity. Therefore, a robust measure to reflect the brain regions where epileptic activity is dominant is needed.

\subsection{Nonparametric Hypothesis Testing}

We further segregate the large regions in the atlas to find localized epileptic regions (largest region and smallest region contain 345 and 10 voxels respectively). K-means clustering is performed on the activity-inducing signals ( $\mathrm{K}$ clusters for regions with number of voxels more than $\mathrm{K} \times 100$ voxels). The EEG IED events are convolved with a Gaussian filter $(\mathrm{FWHM}=3 \mathrm{~s})$ and downsampled to fMRI temporal resolution $\mathrm{TR}=1.5 \mathrm{~s}$. 


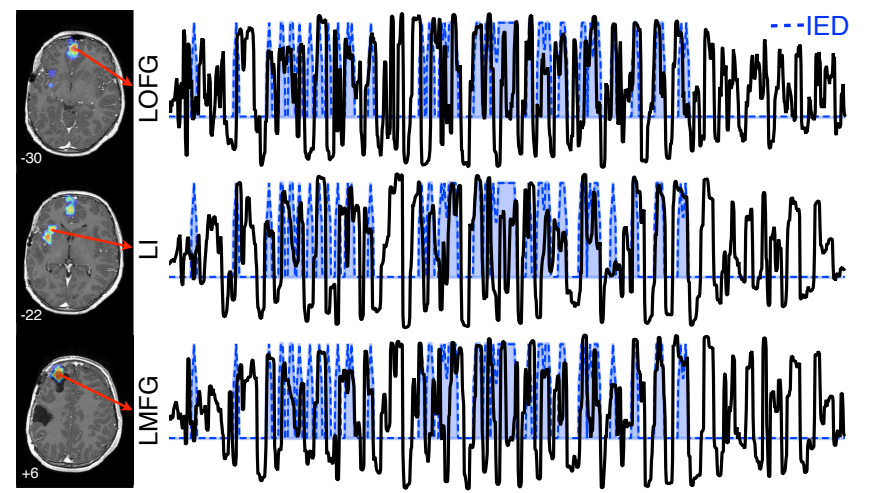

Fig. 2: The ranked average activity-inducing signals (Spearman's correlation) in the selected regions and EEG driven IEDs (blue).

We perform a non-parametric hypothesis testing using correlation (Spearman) of region averaged time courses and original IED. Correlations with 999 surrogates are computed for null hypothesis and fifth highest value of maximum statistics is selected as a threshold ( $p<0.05$ (corrected)). Left Middle Frontal Gyrus (LMFG), Left Medial OrbitoFrontal Gyrus (LOFG), Left Insula (LI) are found to be the significant areas. Among these regions LMFG is already known to be resected in the brain by the experts due to high epileptic activity. Figure 2 shows the ranked average activity-inducing signals in these regions compared with the EEG driven IED onsets.

\subsection{Comparison to Conventional Methods}

The patient was investigated by conventional methods; i.e., feeding EEG information as regressors in GLM analysis (SPM) where the onset of IEDs are defined by an expert during EEG-fMRI [12]. Our method is concordant with the conventional method by locating LMFG as one of the highest possible cortical source of epilepsy. Figure 1 (box III) shows the epileptic regions determined by TA and GLM analysis.

Guided by these non-invasive estimations intracerebral electrodes are placed to the most probable sources of IEDs where recordings determine the areas to be resected during the surgery. The patient had been operated for LMFG, also detected by TA and conventional methods, and left inferior frontal gyrus, not detected by both methods. The patient is reported to be seizure-free after the surgery.

\section{DISCUSSION AND CONCLUSION}

Recently, we have proposed a spatiotemporal regularization method for detecting unpredicted neuronal activity in fMRI. Total activation recovers activity-inducing signals by incorporating a generalized total variation constraint temporally, and by imposing structuredsparsity through an anatomical atlas spatially. The flexibility of the method brings along the adaptation of different type of driving signals by altering the general derivative operator; i.e., $L=L_{h}$ solves for spike-like activity (state-of-the art methods), and the adaptation of a different atlas. TA proved to reconstruct back the paradigm information along with relevant resting state networks in our previous study [7]. Here, we showed that TA is able to locate the cerebral sources of IED taking into account fMRI as its primer modality. The located regions are also confirmed by the conventional methods performed prior to the surgery and intracranial measurements.

\section{REFERENCES}

[1] J. Gotman, E. Kobayashi, A. P. Bagshaw, C.-G. Bénar, and F. Dubeau, "Combining EEG and fMRI: A multimodal tool for epilepsy research," Journal of MRI, vol. 23, no. 6, pp. 906-920, 2006.

[2] F. Moeller, P. LeVan, and J. Gotman, "Independent component analysis (ica) of generalized spike wave discharges in fMRI: Comparison with general linear model-based EEG-fMRI," HBM, vol. 32, no. 2, pp. 209$217,2011$.

[3] L. Hernandez-Garcia and M. O. Ulfarsson, "Neuronal event detection in fMRI time series using iterative deconvolution techniques," $M R I$, vol. 29 , no. 3 , pp. $353-364,2011$.

[4] C. C. Gaudes, N. Petridou, I. L. Dryden, L. Bai, S. T. Francis, and P. A. Gowland, "Detection and characterization of single-trial fMRI BOLD responses: Paradigm free mapping," HBM, vol. 32, no. 9, pp. 1400$1418,2011$.

[5] C. C. Gaudes, N. Petridou, S. T. Francis, I. L. Dryden, and P. A. Gowland, "Paradigm free mapping with sparse regression automatically detects single-trial functional magnetic resonance imaging blood oxygenation level dependent responses," HBM, 2011, in press.

[6] I. Khalidov, J. Fadili, F. Lazeyras, D. Van De Ville, and M. Unser, "Activelets: Wavelets for sparse representation of hemodynamic responses," Signal Processing, vol. 91, no. 12, pp. 2810-2821, 2011.

[7] F. I. Karahanoglu, C. C. Gaudes, and D. Van De Ville, "Total activation: A novel spatio-temporal deconvolution method for fMRI data," Neuroimage, accepted, 2013.

[8] F. I. Karahanoglu, I. Bayram, and D. Van De Ville, "A signal processing approach to generalized 1-d total variation," IEEE TSP, vol. 59, no. 11, pp. $5265-5274$, nov. 2011

[9] H. Raguet, J. Fadili, and G. Peyré, "Generalized forward-backward splitting," 2012, arxiv.org/abs/1108.4404v3.

[10] P.L. Combettes, "Iterative construction of the resolvent of a sum of maximal operators," Convex Analysis, vol. 16, pp. 727-748, 2009.

[11] R. Lopes, J.M. Lina, F. Fahoum, and J. Gotman, "Detection of epileptic activity in fMRI without recording the EEG," NeuroImage, vol. 60, no. 3, pp. $1867-1879,2012$.

[12] F. Grouiller, R.C. Thornton, K. Groening, L. Spinelli, J.S. Duncan, K. Schaller, M. Siniatchkin, L. Lemieux, M. Seeck, C.M. Michel, and S. Vulliemoz, "With or without spikes: localization of focal epileptic activity by simultaneous electroencephalography and fMRI," Brain, vol. 134 , no. 10 , pp. 2867-2886, 2011.

[13] T. E. Lund, K. H. Madsen, K. Sidaros, W. L. Luo, and T. E. Nichols, "Non-white noise in fMRI: Does modelling have an impact?," Neuroimage, vol. 29, no. 1, pp. 54-66, Jan 2006

[14] K. J. Friston, A. Mechelli, R. Turner, and C. J. Price, "Nonlinear responses in fMRI: The balloon model, volterra kernels, and other hemodynamics," NeuroImage, vol. 12, no. 4, pp. 466 - 477, 2000.

[15] J.-C. Baritaux, K. Hassler, M. Bucher, S. Sanyal, and M. Unser, "Sparsity-driven reconstruction for FDOT with anatomical priors," IEEE TMI, vol. 30, no. 5, pp. 1143-1153, May 2011.

[16] A. Beck and M. Teboulle, "Fast gradient-based algorithms for constrained total variation image denoising and deblurring problems," IEEE TIP, vol. 18, no. 11, pp. 2419-2434, Nov. 2009.

[17] N. Tzourio-Mazoyer, B. Landeau, D. Papathanassiou, F. Crivello, O. Etard, N. Delcroix, B. Mazoyer, and M. Loliot, "Automated anatomical labeling of activations in spm using a macroscopic anatomical parcellation of the mni mri single-subject brain," NeuroImage, vol. 15, pp. 273-289, 2002

[18] Y. Alemán-Gómez, L. Melie-Garćia, and P. Valdés-Hernandez, "Ibaspm: Toolbox for automatic parcellation of brain structures," in OHBM'06, 2006, vol. 27

[19] F. Grouiller, L. Vercueil, A. Krainik, C. Segebarth, P. Kahane, and O. David, "A comparative study of different artefact removal algorithms for eeg signals acquired during functional mri," NeuroImage, vol. 38 , no. 1 , pp. $124-137,2007$.

[20] A. Chambolle, "An algorithm for total variation minimization and applications," Journal of Mathematical Imaging, vol. 20, no. 1, pp. 89-97, January 2004. 